\title{
INSTRUMENTOS UTILIZADOS PARA LA MEDICIÓN DE LA CALIDAD DE VIDA RELACIONADA CON LA SALUD DURANTE EL CLIMATERIO
}

Félix Dasio Ayala Peralta ${ }^{1}$, Miguel Angel Rodriguez Herrera², Humberto Izaguirre Lucano ${ }^{3}$, Richard Ayala Palomino ${ }^{4}$, Luis Alberto Quiñones Vásquez ${ }^{5}$, Dasio Ayala Moreno ${ }^{6}$, Antonio Mambret Luna Figueroa ${ }^{7}$,

César Carranza Asmat ${ }^{8}$, Jovanna Ochante Rementería ${ }^{9}$.

\begin{abstract}
RESUMEN
La evaluación de la calidad de vida se debe centrar, principalmente, en la percepción de la paciente sobre su estado de salud, siendo ella partícipe activa en este proceso. Los instrumentos para medir la calidad de vida relacionada con la salud (CVRS) se pueden clasificar en instrumentos genéricos, que miden múltiples áreas o dominios y que son aplicables a todos los individuos, independientemente de la patología que les afecte; y en instrumentos específicos, que son los que miden el funcionamiento del paciente en una o varias áreas y se caracterizan por prestar especial atención a aquellos síntomas o problemas propios de una enfermedad concreta y a aquellas áreas que se consideran más afectadas, siendo sólo aplicables a pacientes específicas. En la práctica clínica diaria es útil para evaluar: mejoría del diagnóstico clínico, individualización y priorización de los tratamientos, mejorar la relación médico-paciente, y monitorizar el cumplimiento de las terapias que contribuyan en la mejora de la calidad de vida femenina.
\end{abstract}

Palabras clave: Calidad de vida; climaterio; instrumentos de evaluación (Fuente: DeCS BIREME).

\section{INSTRUMENTS USED FOR THE MEASUREMENT OF QUALITY OF LIFE RELATED TO HEALTH DURING THE CLIMACTERIC}

\begin{abstract}
The evaluation of quality of life should focus mainly on the patient's perception of her state of health, being an active participant in this process. Instruments for measuring health-related quality of life (HRQL) can be classified into generic instruments that measure multiple areas or domains and that are applicable to all individuals, regardless of the pathology that affects them; And specific instruments, which are those that measure the functioning of the patient in one or several areas and are characterized by paying particular attention to those symptoms or problems specific to a particular disease and those areas considered to be most affected, being only applicable to patients Specific. In daily clinical practice it is useful to evaluate: improvement of clinical diagnosis, individualization and prioritization of treatments, improving the doctor-patient relationship, and monitoring compliance with therapies that contribute to the improvement of the female quality of life.
\end{abstract}

Key words: Quality of life; Climacteric; Evaluation tools (Source:MeSH NLM).

\section{INTRODUCCIÓN}

El climaterio es una etapa de la vida de la mujer donde se producen una declinación paulatina y cese de la función ovárica, que es un proceso que acontece en años. En este lapso de tiempo muchas mujeres experimentan síntomas climatéricos que deterioran la calidad de vida de manera importante y presentan incremento de los factores de riesgo de enfermedades crónicas. Para ello, es pertinente la medición de la calidad de vida que nos permita adoptar una conducta terapéutica consensuada en la mujer. En aquellas que las requieran, se debe realizar alguna intervención médica como cambios de estilos de vida, terapia hormonal, hipolipemiante, antiresortivo, entre otras. La menopausia no determina la indicación de terapia; lo hacen el deterioro de la calidad de vida y los riesgos de enfermedades crónicas ${ }^{1}$.

Médico GínecoObstetra. Magíster en Salud Reproductiva. Profesor de la Facultad de Medicina Humana de la Universidad San Martín de Porres y de Ciencias de la Salud de la Universidad Norbert Wiener. Jefe del Servicio de Climaterio y Menopausia del Instituto Nacional Materno Perinatal. Miembro de la Red Latinoamericana de Investigadores en Climaterio (REDLINC). Lima-Perú.

Médico GínecoObstetra. Ex Residente de Ginecología y Obstetricia de la Universidad Nacional Mayor de San Marcos. Sede Instituto Nacional Materno Perinatal. Ex Director del Hospital II-1 Cangallo, Ayacucho. Lima-Perú.

Médico GínecoObstetra. Jefe del Servicio de Ginecología Patológica del Instituto Nacional Materno Perinatal. Miembro de la Red Latinoamericana de Investigadores en Climaterio (REDLINC). Lima-Perú.

4 Médico Cirujano. Universidad Privada San Juan Bautista. Lima-Perú.

5 Médico GínecoObstetra. Asistente del Servicio de Obstetricia C. Instituto Nacional Materno Perinatal. Lima-Perú.

6 Interno de Medicina de la Facultad de Medicina Humana de la Universidad Nacional Federico Villarreal, Lima-Perú.

7 Médico GínecoObstetra. Profesor Principal de la Universidad Nacional Mayor de San Marcos. Director Ejecutivo de Investigación, Docencia y Atención en Obstetricia y Ginecología del Instituto Nacional Materno Perinatal. Lima-Perú.

8 Médico GínecoObstetra. Profesor Principal de la Universidad Nacional Mayor de San Marcos. Director Ejecutivo de la Oficina de Investigación y Docencia Especializada. Instituto Nacional Materno Perinatal. Lima-Perú.

9 Licenciada en Trabajo Social. Servicio de Trabajo Social. Instituto Nacional Materno Perinatal. Lima-Perú 
La calidad de vida de muchas mujeres climatéricas en todo el mundo ha sido disminuido significativamente tras los informes del estudio Women's Health Initiative (WHI) con el resultado en el uso de la terapia hormonal que ha descendido en el $50 \%$ o más durante los 10 años subsiguientes al estudio. La medición de la calidad de vida es dificultoso porque contribuyen muchos factores y existen numerosos y diferentes instrumentos, algunos de los cuales evalúan la salud en general y sólo unos pocos que específicamente incluyen síntomas relacionados con la menopausia. La TH mejora la calidad de vida de las mujeres menopáusicas sintomáticas y algunos estudios sobre los efectos de la TH proporcionan evidencia confiable sobre la calidad de la vida con la reducción de los síntomas vasomotores ${ }^{2}$

\section{CALIDAD DE VIDA}

El concepto de calidad de vida ha ido evolucionando en el tiempo desde una concepción sociológica hacia una perspectiva psicosocial en la que lo relevante es la satisfacción personal. En 1994, la Organización Mundial de Salud $(\mathrm{OMS})^{3}$ la define como «la percepción que tiene el individuo de su situación en la vida, dentro del contexto cultural y de valores en que vive, y en relación con sus objetivos, expectativas e intereses». En esta definición subyacen dos elementos esenciales: por un lado, su carácter subjetivo, enunciado como un sentimiento personal, un juicio o una comparación; y, por otro, su carácter multidimensionalidad, abarcando aspectos físicos, afectivos, cognitivos, sociales, económicos, entre otras.

En la actualidad, está ampliamente aceptada la multidimensionalidad del concepto de calidad de vida. Sin embargo, cuando una enfermedad afecta a un individuo el deterioro, no solo afecta a él física o emocionalmente, sino que también a su capacidad económica, a su entorno familiar y a veces, incluso, a sus valores religiosos o políticos. Así surge la necesidad de crear el término de Calidad de Vida Relacionada con la Salud (CVRS), que ha sido definida como «la valoración subjetiva del paciente orientada hacia el exterior del mismo, y centrada en el impacto del estado de la salud sobre su capacidad de llevar una vida subjetivamente satisfactoria» ${ }^{4}$

Recientemente, centrada en las necesidades del paciente, se define a la CVRS como: "Nivel de bienestar y satisfacción del individuo, tanto físico como mental y social en relación con los problemas de salud" ${ }^{3,5,6}$.

Los cambios biológicos que se producen durante la transición menopáusica ${ }^{7}$ no sólo provocan irregularidades del ciclo menstrual sino también otras manifestaciones, incluyendo síntomas vasomotores, psicológicos y genitourinarios, que afectan profundamente la calidad de vida ${ }^{8}$.

\section{MEDICIÓN DE LA CALIDAD DE VIDA DURANTE EL CLIMATERIO}

La evaluación de la calidad de vida se debe centrar, principalmente, en la percepción de la paciente sobre su estado de salud, siendo ella partícipe activa en este proceso.

Los instrumentos para medir la calidad de vida relacionada con la salud (CVRS) se pueden clasificar en instrumentos genéricos que son aquellos que miden múltiples áreas o dominios, tales como el funcionamiento físico, social y mental, y que son aplicables a todos los individuos, independientemente de la patología que les afecte; y en instrumentos específicos, que son los que miden el funcionamiento del paciente en una o varias áreas y se caracterizan por prestar especial atención a aquellos síntomas o problemas propios de una enfermedad concreta y a aquellas áreas que se consideran más afectadas, siendo sólo aplicables a pacientes específicos ${ }^{9-11}$.

\section{CUESTIONARIOS GENÉRICOS DE EVALUACIÓN DE CALIDAD DE VIDA}

Los cuestionarios genéricos de calidad de vida exploran la capacidad del individuo en diferentes áreas denominadas dominios. Aunque los dominios varían de una escala a otra, de una manera general, todos los cuestionarios contienen 3 grandes bloques: dominio físico, emocional o afectivo y dominio social. En el dominio físico, se evalúa la presencia de trastornos orgánicos, limitaciones ocasionadas por alguna enfermedad; en el área emocional, se investiga la calidad de vida en el campo psicológico: presencia de tristeza, irritabilidad, ansiedad, entre otras; en el dominio social se explora su integración en la sociedad y su capacidad para el trabajo y la vida diaria ${ }^{6,12}$.

En ocasiones algunos cuestionarios incluyen otro domino denominado genéricamente "vitalidad", donde se explora este aspecto del psiquismo ${ }^{6,13}$.

En tal sentido, las escalas genéricas son multidimensionales y con ellas se puede explorar un amplio abanico de factores que influyen en la calidad de vida. Las herramientas genéricas permiten la comparación entre diferentes patologías, en cuanto la calidad de vida. Sin embrago, en ocasiones son poco sensibles para evaluar la evolución de una determinada enfermedad con diferentes terapéuticas. Uno de los inconvenientes de estas escalas, es su menor sensibilidad y especificidad. Entre las escalas genéricas más usadas se encuentran: Nottingham Health Profile $^{14}$, Short Form-36 (SF-36) ${ }^{15}$, Psicological General Well Being (PGWB) ${ }^{16}$, y la escala europea de calidad de vida (EuroQOL) $)^{6,17}$.

El estudio GAZEL ${ }^{18}$ es una investigación que se realizó en Francia con 286 mujeres de la compañía de gas y electricidad francesa. Se utilizó como instrumento el 
Nottingham Health Profile, un instrumento de calidad de vida general. Este instrumento halló que tras conseguir por edad, la mujer posmenopáusica presentaba peor calidad de vida que la premenopáusica para 4 de las 6 secciones analizadas, concretamente: aislamiento social, dolor, sueño y energía; todos ellos explicables por los trastornos propios del climaterio ${ }^{6}$.

El Short Form-36 (SF-36) es el cuestionario genérico más ampliamente utilizado, contiene 36 ítems que evalúan la calidad de vida relacionada a la salud en 8 áreas o dominios abarcando status funcional, bienestar y evaluación global de salud. Este instrumento de autoevaluación permite medir la percepción de salud que tiene la población en general 14. El SF-36 ha sido utilizado en un gran número de estudios poblacionales y en diferentes condiciones clínicas, mostrando excelentes propiedades psicométricas 19. Ha sido traducido y validado en diferentes idiomas ${ }^{6,20}$.

En una investigación ${ }^{21}$, aplicando el cuestionario SF-36 en 103 mujeres y 86 varones en Londres, encontraron tanto en los varones como en las mujeres similar nivel de CVRS, satisfacción y salud general, aunque las mujeres reportaron más problemas físicos. Asimismo, los factores predictivos de CVRS significativos fueron : enfermedades serias, actividad laboral y status marital.

El Psicological General Well Being (PGWB), es una escala genérica que explora la calidad de vida con un enfoque muy detallado del dominio emocional y psicológico; contiene 22 ítems y evalúan vitalidad, salud, bienestar, autocontrol, ansiedad y depresión ${ }^{16}$.

\section{CUESTIONARIOS ESPECÍFICOS PARA EVALUACIÓN DE CALIDAD DE VIDA RELACIONADA CON LA SALUD DURANTE EL CLIMATERIO}

La utilidad de la valoración de la calidad de vida en el climaterio en la actualidad es imprescindible para el conocimiento y evaluación de medidas preventivas y terapéuticas; así como, para comparar situaciones geográficas y su seguimiento en el tiempo ${ }^{11,22,23}$.

Entre las escalas más importantes de calidad de vida relacionadas con la salud (CVRS) tenemos: Índice de Blatt-Kupperman ${ }^{24}$, el Women Health Questionnaire (WHQ) ${ }^{25}$, el cuestionario MENCAV ${ }^{26}$, la Menopause Rating Scale (MRS) ${ }^{27}$, la Menopause Quality of Life (MENQOL) ${ }^{28,29}$, la Utian Quality of Life Score (UQOL) ${ }^{30}$, la Escala climatérica de Greene ${ }^{31}$ y la Escala Cervantes ${ }^{32}$.

El Índice de Blatt-Kupperman ${ }^{24}$, ha sido el referente tradicional para evaluar el impacto de la terapia hormonal sobre la sintomatología climatérica. En cada síntoma tiene asignado una constante que se multiplica por el valor otorgado a la intensidad de la sintomatología; dicha intensidad se valora en: severa ( $>35)$, moderada
( 20-35) y leve $(<20)$. Sin embargo, esta escala no refleja necesariamente cómo las mujeres perciben sus síntomas o responden al tratamiento y ha sido criticado recientemente en la literatura médica ${ }^{27,33}$.

EI Women Health Questionnaire ( WHQ $)^{25}$ es un cuestionario desarrollado en Inglaterra en 1992, consta de 36 ítems reunidos en 9 subescalas que describen: síntomas somáticos ( 7 ítems), humor depresivo ( 7 ítems), dificultades en memoria/concentración ( 3 ítems) , ansiedad/miedos ( 4 ítems), comportamiento sexual (3 ítems), síntomas vasomotores (2 ítems), trastornos del sueño ( 3 ítems), trastornos menstruales ( 4 ítems) y atracción ( 2 ítems). A la intensidad de la sintomatología se le otorga un valor de 1 a 4 según la severidad de la misma, cuanto más alto es el puntaje, más pronunciado es el distrés y la disfunción.

Este cuestionario es ampliamente aplicado en área de la menopausia, validado y utilizado para evaluar los cambios inducidos por diferentes tratamientos ${ }^{6}$.

La Menopause Quality of Life (MENQOL) ${ }^{28,29}$, es un instrumento especialmente diseñado para evaluar la calidad de vida en el climaterio, desde la perspectiva propia de las mujeres. Este cuestionario específico de calidad de vida para la menopausia ha demostrado poseer todas las cualidades psicométricas necesarias: fiabilidad, validez y sensibilidad.

Hilditch et $\mathrm{al}^{28}$ propusieron un acercamiento distinto directamente orientado a la calidad de vida desde su inicio. El resultado de esta investigación fue el cuestionario específico de calidad de vida, conocida por su sigla inglesa MENQOL (Menopause Quality of life), donde se identifican 29 síntomas agrupados en cuatro dominios: vasomotor, psicosocial, físico y sexual. Se explora la intensidad del síntoma; percibido colocando una puntuación entre cero cuando no le molesta nada; y 6 , si es que le molesta mucho. Para el cálculo del puntaje se da: Si la respuesta es No, se asigna un puntaje de 1; si la respuesta es Sí y 0 , se signa 2; si es Sí y 1 asigne 3 y así sucesivamente hasta Sí y 6 , se le asigna 8 . Se saca el puntaje promedio de cada área. No existe un puntaje global, sino que cada área en particular (anexo $\mathrm{A}$ ). Éste es un instrumento especialmente concebido para valorar calidad de vida en el climaterio, desde la perspectiva propia de las mujeres .

Blümel et al ${ }^{29}$, tras incluir 481 mujeres entre 40 y 59 años, utilizando el instrumento específico de calidad de vida para la menopausia de Hilditch, encontró un deterioro considerable en la calidad de vida de las mujeres climatérica, con importantes diferencias respecto a las premenopáusicas. Los cuatro dominios de síntomas se deterioran cuando comienzan las irregularidades menstruales en la perimenopausia, no observándose cambios significativos cuando posteriormente cesan los flujos menstruales; el daño de calidad de vida es previa a la menopausia. Al aplicar regresión logística, observaron 
que la pérdida de calidad de vida en estas mujeres no dependía de la edad ni de variables sociodemográficas como el estado civil, número de hijos, educación o actividad laboral, sino de su status hormonal subsecuente a la menopausia.

Ortiz y $\mathrm{col}^{34}$, utilizando el MENQOL de Hilditch, encontró que los síntomas vasomotores que seleccionó Hilditch como propios del climaterio en mujeres americanas también aparecen en las colombianas, pero no observando igual concordancia con los síntomas psicosociales. Este estudio no encontró que se incremente durante el climaterio la tristeza, el nerviosismo, la impaciencia o el descontento con la vida personal; pero sí, la pérdida de memoria, el deseo de soledad y el sedentarismo. Igualmente encontró discrepancias en varias manifestaciones físicas y sexuales encontradas por Hilditch. Estas observaciones plantean dudas en cuanto a lo adecuado o no de usar escalas desarrolladas en un país distinto al cual se aplica dicha escala.

Ayala y $\mathrm{col}^{35}$ en la unidad de climaterio, del Instituto Nacional Materno Perinatal de Lima, realizó un estudio de 110 pacientes climatéricas peruanas comprendidas entre 30 y 75 años de edad, aplicando el cuestionario específico MENQOL de Hilditch. El 49\% de pacientes estuvieron en la posmenopausia natural, el $17 \%$ en la premenopausia, el $18 \%$ en la perimenopausia y el $16 \%$ conformaron la menopausia quirúrgica. Los síntomas más frecuentes fueron los psicofísicos, seguido de síntomas sexuales y con menos frecuencia los vasomotores. En cuanto al impacto del climaterio, se observa que, conforme avanzan las etapas del climaterio, se deteriora la calidad de vida de la mujer, sobre todo en las áreas psicosocial y físico (tabla 1). Resultado similar ${ }^{37}$ se obtuvo al aplicar la escala climatérica de Greene.

Tabla 1. Impacto del climaterio en la calidad de vida.

\begin{tabular}{lcccc}
\hline \multicolumn{1}{c}{ IMPACTO } & VASOMOTOR PSICOSOCIAL & FíSICO & SEXUAL \\
\hline Premenopausia & $2 \pm 0,99$ & $3,53 \pm 1,45$ & $3,11 \pm 1,05$ & $2,66 \pm 1,58$ \\
Perimenopausia & $2,9 \pm 1,46$ & $4,64 \pm 1,52$ & $4,14 \pm 1,32$ & $2,95 \pm 1,97$ \\
$\begin{array}{l}\text { Posmenopausia } \\
\text { natural }\end{array}$ & $2,55 \pm 1,58$ & $4,14 \pm 1,46$ & $4,01 \pm 1,12$ & $3,33 \pm 2,07$ \\
$\begin{array}{l}\text { Posmenopausia } \\
\text { quirúrgica }\end{array}$ & $2,27 \pm 1,38$ & $3,60 \pm 1,71$ & $3,86 \pm 1,15$ & $3,59 \pm 2,20$ \\
$\mathbf{E}<$ & 0,0001 & 0,0001 & 0,0001 & 0,0001 \\
\hline
\end{tabular}

La Escala climatérica de Greene ${ }^{31}$, es un instrumento que ha intentado construirse a partir de la medición de síntomas climatéricos basada en principios científicos objetivos.

Uno de los avances importantes en la evaluación del climaterio es esta escala que fue desarrollada de acuerdo a la metodología de análisis factorial, donde esencialmente se analiza largas series de síntomas, a fin de establecer qué relaciones existen entre ellos, y así poder formar grupos o dominios. De esta forma se consigue agrupar síntomas. Además, la técnica permite establecer un coeficiente de correlación entre síntoma y dominio, de modo que, según la magnitud de ese coeficiente, se puede medir la fortaleza de la asociación entre ambos. Los 21 síntomas climatéricos seleccionados contiene 3 puntuaciones cada una, que la paciente refiere percibir según la intensidad de la molestia desde 0: ninguna molestia; 1: que indica poca molestia;2: una molestia regular; y 3: le molesta mucho. Todos ellos caen en 4 dominios o grupos independientes: vasomotor, psicológico, somático y sexual.

En un estudio realizado en la población ecuatoriana ${ }^{36}$ de 385 mujeres de 40 a 65 años valoradas con la Escala de Greene, se encontró en altos porcentajes dificultad en la concentración (87\%), sentirse infeliz (82\%), cefalea $(83,9 \%)$ y sofocos $(82 \%)$; y al aplicar el análisis multivariado determinó que la edad, la menor educación y la mayor paridad fueron los factores involucrados de presentar mayores puntajes de la sub-escala psicológica, somática y sexual, respectivamente. Concluyen que la Escala de Greene es confiable, permite el análisis factorial, pero fue básicamente diseñada, si bien por dominios para evaluar síntomas climatéricos, pero no constituye un buen instrumento para evaluar calidad de vida.

En la investigación realizada por Ayala $\mathrm{F}^{37}$ con la aplicación de la escala climatérica de Greene en 196 mujeres climatéricas entre 35 y 65 años de edad se evidencia que las cuatro áreas se deterioran cuando se inicia la premenopausia, y conforme avanza las etapas del climaterio, se incrementa éste deterioro, sobre todo en las áreas psicológica, vasomotor y somática; predominan en la perimenopausia y persiste en la postmenopausia. Con respecto al área somática, se nota que el deterioro se mantiene relativamente constante, pero en menor escala en las diferentes etapas del climaterio, predominando en la perimenopausia. En cambio, el deterioro del área sexual es más frecuente durante la etapa de la postmenopausia. Concluye que al aplicar la Escala de Greene en el climaterio nos sugiere que existe un deterioro considerable en la calidad de vida relacionada a la salud femenina, sobre todo en las áreas psicológica y somática, que parece depender, principalmente de la deficiencia estrogénica.

La Menopause Rating Scale (MRS) ${ }^{27,38}$, es una escala bien definida de autoevaluación de los síntomas menopáusicos, consta de 11 ítems, que permitirá de una manera práctica y relativamente rápida evaluar el impacto de cualquier intervención médica en relación a diferentes aspectos de la calidad de vida ${ }^{6}$ (anexo B).

LaMRSesuncuestionarioestandarizadodeautoevaluación integrado por 11 ítems, que fue confeccionado en 1994 por un grupo de expertos de las Sociedades de Menopausia Alemana, Suiza y Austraica ${ }^{38}$. Esta escala fue revisada y validada en 1996 en base a un estudio realizado con una 
muestra representativa randomizada con 683 mujeres alemanas de 45 a 60 años de edad que completaron la escala MRS ${ }^{39}$. Un seguimiento luego de 18 meses con la misma población de mujeres revalidó nuevamente la escala variando la puntuación original. Las mujeres respondedoras de MRS modificada deben elegir entre 5 grados de severidad: asintomáticas ( 0 puntos), leve (1 punto), moderado ( 2 puntos), severo (3 puntos) e intenso (4 puntos); a cada uno de los 11 síntomas se le asigna un puntaje, obteniendo un score total de MRS que oscila entre 0 ( asintomática) y 44 ( grado máximo de disturbio por síntomas menopáusicos $)^{40}$.

La utilización en los últimos años de la Menopause Rating Scale (MRS), un instrumento específico para evaluar la sintomatología climatérica, por muchos investigadores $y$ en diversos lugares del mundo ha sido un avance importante que nos permite objetivar y comparar los efectos clínicos del climaterio en diversas poblaciones ${ }^{41}$.

El grupo REDLINC ${ }^{42}$ ha realizado un gran estudio con el objetivo de valorar la calidad de vida de la mujer Latinoamericana de mediana edad, donde enrolaron 8,373 mujeres de 40 a 59 años donde evaluaron con la escala de puntuación en menopausia (MRS), y un cuestionario general (datos personales y del compañero sexual). Este estudio cubrió mujeres de 18 ciudades en 12 países de América Latina donde se aprecia un marcado deterioro de la calidad de vida de la mujer en Latinoamérica, siendo mayor en unos países con relación a otros; como Chile con el 80,8\%; Ecuador con el 60,1\%; Perú con el 51,6\%; Colombia $48,3 \%$, entre otras, y el promedio latinoamericano de $55,4 \%{ }^{42}$. En el análisis de regresión logística asociados a mayores puntajes totales del MRS (deterioro de la calidad de vida) fueron: socio demográficos: vivir en la altura (OR 1,14); femeninos: uso de alternativas para la menopausia (OR 1,85) y psicotrópicos (OR 1,75); masculino: eyaculación precoz (OR 1,84). Asimismo, cuando se analizó por dominios y se comparó entre países y la región (como por ejemplo el dominio psicológico), Chile rebasa ampliamente el promedio Latinoamericano y de Perú ( 7,7 versus 4,6 y 4,3 respectivamente, de puntaje total del dominio psicológico). Igualmente el dominio somático, con un puntaje total para Chile de 16,3 versus 11,3 para América Latina y 3,5 para Perú.

Por otro lado, en otro estudio se pudo apreciar que la etnicidad también influye. En efecto, las mujeres Colombianas afrodescendientes presentaron mayor severidad de la sintomatología psicológica y somática que las mestizas ${ }^{43,44}$. Igualmente las mujeres indígenas del altiplano peruano (Quechua) presentaron mayor sintomatología que indígenas de la costa caribeña de Colombia (Xenú) ${ }^{45}$.

EI MRS es un instrumento confiable para evaluar calidad de vida para la práctica clínica, por ser corto (11 ítems) y fácil de aplicar. Algunos han objetado su capacidad para evaluar respuesta a la terapia hormonal. Asimismo, otros estudios permiten apreciar que en América Latina la terapia hormonal mejora la calidad de vida ${ }^{42,46}$.

La Utian Quality of Life Score (UQOL) ${ }^{30}$, es un cuestionario de calidad de vida en la mujer perimenopáusica que está fuertemente basado en la percepción de la sensación de bienestar. Consiste en un instrumento de 23 ítems agrupados en 4 dominios , validado con las escalas genéricas de calidad de vida en una muestra de mujeres perimenopáusicas de diferentes ámbitos geográficos. Los diseñadores de la UQOL estudiaron 597 mujeres (edad media de 52,9 años) de 11 comunidades norteamericana. La UQOL demostró propiedades psicométricas adecuadas en los 4 dominios, denominadas ocupacional, salud, emocional y sexual. Esta escala resulta una herramienta útil en la valoración de la calidad de vida de la mujer menopáusica para la investigación clínica y la práctica médica ${ }^{6}$.

La Escala Cervantes ${ }^{32}$, ha sido desarrollada y validada en España que permite valorar la calidad de vida y evaluar la intervención terapéutica. Su estructura ha quedado definitivamente en 31 ítems estructurados en las 4 dimensiones de: menopausia y salud, sexualidad, dominio psíquico y relación de pareja. La dimensión de menopausia y salud incluyen las subdimensiones de sintomatología vasomotora, salud y envejecimiento. Este instrumento específico para la mujer española durante la perimenopausia y la postmenopausia, es capaz de valorar el impacto de su estado de salud en distintas áreas físicas y psicosociales $\mathrm{y}$, en especial, su repercusión en el bienestar general. Se trata del primer cuestionario que, por haber sido desarrollado y validado en una muestra representativa de 2274 mujeres de la población española, permite su uso generalizado en la población femenina de 45 a 64 años de edad ${ }^{6,32}$.

\section{UTILIDAD CLÍNICA DE UNA ESCALA DE MEDICIÓN DE CALIDAD DE VIDA DURANTE EL CLIMATERIO}

La medición de la calidad de vida se justifica plenamente con instrumentos apropiados, por cuanto mejoran la comprensión de los problemas que atraviesa la mujer en esta etapa de la vida desde un punto de vista biopsicosocial, y por tanto un adecuado manejo de los síntomas.

La utilidad clínica radica en que una escala nos permite conocer y evaluar la calidad de vida durante el climaterio, dando a conocer la realidad en un momento dado, en una situación geográfica o en una población determinada. Además, pueden compararse entre sí, con otras razas o etnias, situaciones económicas diferentes, con la misma población en un tiempo diferente, etc. Es decir, en la clínica diaria es útil para evaluar: mejoría del diagnóstico clínico; individualización y priorización de los tratamientos; 
mejorar la relación médico-paciente; monitorización del tratamiento ; y mejoría del cumplimiento de las terapias. Asimismo, otro parámetro interesante de evaluar es el impacto de un determinado tratamiento, dosis, pautas, entre otras, sobre la calidad de vida. Hoy en día estas escalas de calidad de vida se han convertido en una necesidad que nos permita monitorizar la mantención o mejoría en lo posible de la calidad de vida del paciente. Por, ello la utilidad de la valoración de la calidad de vida en investigación clínica, incluyen: en el entorno del climaterio la consideración del climaterio con una perspectiva global; evaluación del impacto de una determinada terapéutica, efecto dosis o efectividad de las combinaciones terapéuticas; desarrollo de nuevas moléculas; y la calidad de vida como análisis obligado ${ }^{6,32}$.

\section{FACTORES QUE INFLUYEN EN LA CALIDAD DE VIDA DURANTE EL CLIMATERIO}

La menopausia es un fenómeno biopsicosociocultural, en donde los factores biológicos, psicológicos y socioculturales interactúan en la expresión de la vivencia durante el climaterio y determinan su impacto en la calidad de vida de la mujer.

En la literatura se relaciona la intensidad de los síntomas climatéricos con la presencia de condiciones biopsicosociales, que pueden modular la calidad de vida ${ }^{47,48}$. Entre estos factores, destacan la edad y el nivel sociocultural, características que han sido consideradas factores de riesgo independientes para la severidad de la sintomatología climatérica ${ }^{49}$.

La etnia es otro factor que puede afectar a la prevalencia de la sintomatología climatérica en las distintas poblaciones 50,51 . Así, en un estudio en las mujeres colombianas de raza negra tenían más deterioro de calidad de vida durante el climaterio que las mujeres hispánicas, lo cual era debido fundamentalmente a una mayor severidad de los síntomas psicológicos y somáticos ${ }^{43}$; mientras que las indígenas presentan más sintomatología del área urogenital ${ }^{44}$.

Asimismo, en un estudio multiétnico realizado en 7 regiones de Estados Unidos con 16065 mujeres de 40-55 años de edad, mostraron que las hispánicas presentan con más frecuencia incontinencia urinaria y sequedad vaginal que las mujeres pertenecientes a las etnias blancas, caucásicas, chinas, japonesas y afroamericanas ${ }^{52}$.

La severidad de los síntomas climatéricos es muy diferente según los estratos socioeconómicos, así una mujer sin profesión presentará más síntomas que la profesional ${ }^{53}$.

Por otro lado, el impacto que tiene el climaterio en la calidad de vida es atribuida a la sintomatología asociada, especialmente los vasomotoras que provocan en algunas mujeres grandes molestias diurnas e interrumpen el sueño ${ }^{54}$; así como los síntomas urogenitales pueden interferir con las actividades cotidianas, los eventos sociales, laborales y repercutir también en la esfera psicosexual ${ }^{55}$.

Asimismo, durante el climaterio muchas mujeres manifiestan alteraciones psicosomáticas, sin embargo la menopausia per se no tendría efecto deletéreo en la salud mental; ya que muchos de los trastornos descritos se vinculan más a factores psicosociales y su relación directa con la caída estrogénica es poco probable ${ }^{56}$; ya que también influyen otros factores tales como los aspectos genéticos y el envejecimiento mismo ${ }^{57}$. 


\section{ANEXO A. CUESTIONARIO ESPECÍFICO DE CALIDAD DE VIDA EN LA MENOPAUSIA DE HILDITCH}

I. FILIACIÓN :

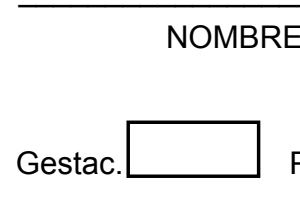

Anticoncep. Actual

Paridad

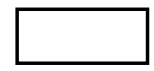

F.U.R.

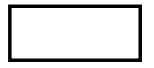
Edad de la menopausia Sí ¿cuál?
FECHA:

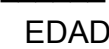

PROCEDENCIA

Menstrúa No

Sí ¿desde cuándo?

Regular

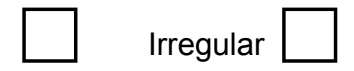

Ciclos largos

Ciclos cortos

\section{ANTECEDENTES PATOLÓGICOS:}

ANTECEDENTES QUIRÚRGICOS:

\section{DIAGNÓSTICO ACTUAL:}

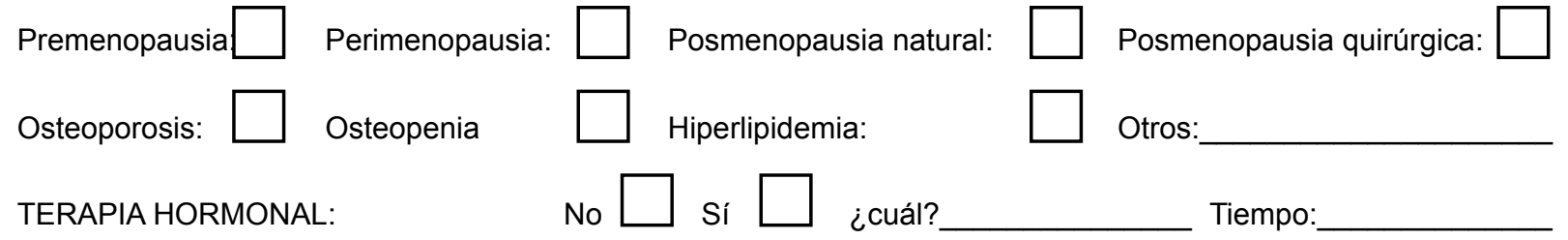

II. Instrucciones: Lea completo todo el texto. No se salte líneas. Cada una de las preguntas viene en la siguiente forma:
1. ¿Tiene bochornos?
No
Sí

Si Ud. no ha tenido el problema en el último mes

Marque "NO"

Si Ud. ha tenido el problema en el último mes

Marque "SI"

y ponga nota a la intensidad de las molestias

No molesta

0
Molesta mucho

$\begin{array}{llllll}1 & 2 & 3 & 4 & 5 & 6\end{array}$

PARA CADA UNADE LAS SIGUIENTES PREGUNTAS, INDIQUE SI UD. HA SENTIDO O TENIDO LA MOLESTIA EN EL ÚLTIMO MES. SI LA HA TENIDO COLOQUE UNA NOTA A LA INTENSIDAD DE LA MOLESTIA ENTRE 0 (NO LE MOLESTA NADA) Y 6 (MOLESTA MUCHO).
1. ¿Tiene bochornos?

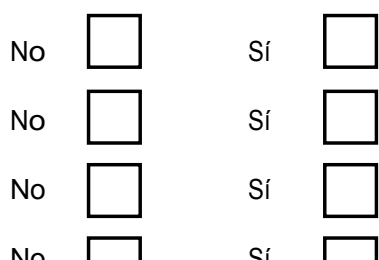
0
$\begin{array}{llllll}1 & 2 & 3 & 4 & 5 & 6\end{array}$
2. ¿Transpiraciones nocturnas?
0
$\begin{array}{llllll}1 & 2 & 3 & 4 & 5 & 6\end{array}$
3. ¿Más transpiración que lo normal?
No
Sí
0
$\begin{array}{llllll}1 & 2 & 3 & 4 & 5 & 6\end{array}$
0
$\begin{array}{llllll}1 & 2 & 3 & 4 & 5 & 6\end{array}$ 

5. ¿Nerviosismo o ansiedad?
$\begin{array}{ll}\text { No } & \square \\ \text { No } & \square \\ \text { No } & \square \\ \text { No } & \square \\ \text { No } & \square \\ \text { No } & \square \\ \text { No } & \square \\ \text { No } & \square\end{array}$

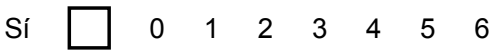
6. ¿Pérdida de memoria?
Sí $\square \quad \begin{array}{lllllll}0 & 1 & 2 & 3 & 4 & 5 & 6\end{array}$
7. ¿Hace menos cosas de las que acostumbraba?
Sí $\square \quad \begin{array}{lllllll}0 & 1 & 2 & 3 & 4 & 5 & 6\end{array}$
8. ¿Se siente deprimida o triste?
Sí $\square=0 \begin{array}{lllllll} & 0 & 2 & 3 & 4 & 5 & 6\end{array}$
9. ¿Es impaciente con las otras personas?
Sí $\square \quad \square \quad \begin{array}{lllllll} & 0 & 2 & 3 & 4 & 5 & 6\end{array}$
10. ¿Siente necesidad de estar sola?

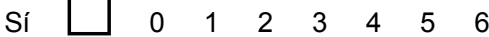
11. ¿Tiene flatulencia, gases o hinchazón abdominal?
Sí $\square 0 \begin{array}{lllllll}0 & 1 & 2 & 3 & 4 & 5 & 6\end{array}$
12. ¿Tiene dolores osteomusculares y/o articulares?

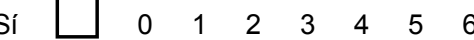
13. ¿Se siente cansada o agotada físicamente?
No
14. ¿Tiene dificultad para dormir?
15. ¿Tiene dolores de cabeza o de nuca?
16. ¿Tiene disminución de la fuerza física?
17. ¿Tiene disminución de l a resistencia física?
18. ¿Tiene sensación de falta de energía?
19. ¿Tiene sequedad de la piel?
20. ¿Tiene aumento de peso?
21. ¿Tiene aumento de pelos en la cara?
22. ¿Cambios en la piel como arrugas o manchas?

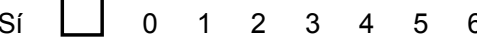
23. ¿Retiene líquido?
24. ¿Tiene dolor de cintura?
25. ¿Orina frecuentemente?
26. ¿Se orina al reirse, toser, o hacer un esfuerzo?
27. ¿Ha tenido cambios en su deseo sexual?
28. ¿Tiene resequedad vaginal durante el acto sexual?

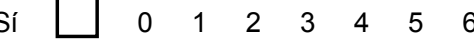
No

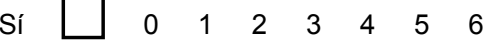
$\begin{array}{ll}\text { No } & \square \\ \text { No } & \square \\ \text { No } & \square \\ \text { No } & \square\end{array}$

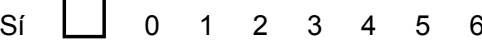
Sí $\square \quad 0 \quad 1 \quad 2 \quad 3 \quad 3 \quad 4 \quad 5 \quad 6$
Sí $\square \quad \begin{array}{lllllll}0 & 1 & 2 & 3 & 4 & 5 & 6\end{array}$

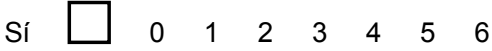
No

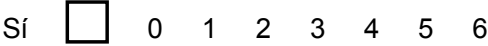
Sí $\square=0 \begin{array}{lllllll} & 0 & 2 & 3 & 4 & 5 & 6\end{array}$
No $\square$

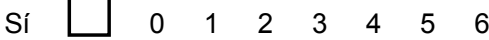
No

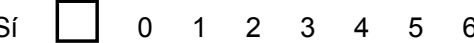
29. ¿Evita las relaciones sexuales?
30 ¿Siente dolor durante la relación sexual?

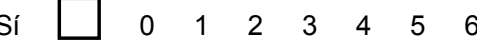
Sí $\square=0 \begin{array}{lllllll} & 0 & 2 & 3 & 4 & 5 & 6\end{array}$
Sí $\square \quad \begin{array}{lllllll}0 & 1 & 2 & 3 & 4 & 5 & 6\end{array}$

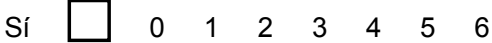
Sí $\square \quad \begin{array}{lllllll}0 & 1 & 2 & 3 & 4 & 5 & 6\end{array}$

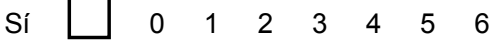

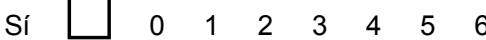

PUNTAJE. Si la respuesta es NO, asigne un puntaje de 1; si la respuesta es Sí y 0, asigne 2; si es Sí y 1, asigne 3; y así sucesivamente hasta Sí y 6, que se le asigna 8. El test se divide en 4 áreas: Vasomotora, preguntas 1 a 3; Psicosocial, preguntas 4 a 10; Física, preguntas 11 a 26; y Sexual, preguntas 27 a 30 . Se saca puntaje promedio de cada área. No existe un puntaje global, sino el de cada área en particular.. 


\section{ANEXO B. ESCALA DE PUNTUACIÓN EN MENOPAUSIA (MRS) Menopause Rating Scale}

Con el objeto de conocer sus molestias climatéricas le solicitamos responder el siguiente cuestionario marcando el casillero correspondiente

¿Cuál de la siguientes molestias siente en la actualidad y con qué intensidad?

\begin{tabular}{|c|c|c|c|c|c|c|c|}
\hline \multirow[b]{2}{*}{ 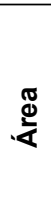 } & \multirow[b]{2}{*}{$\stackrel{\Xi \Phi}{\Phi}$} & \multirow{2}{*}{$\begin{array}{l}\text { TIPO DE MOLESTIAS } \\
\text { (Marque la casilla pertinente de cada molestia con una "X". } \\
\text { Ejemplo, marque en casilla } 0 \text { cuando "no tiene molestia" y } \\
\text { en la casilla } 1 \text { a la } 4 \text { según como sienta la intensidad de la } \\
\text { molestia). }\end{array}$} & \multicolumn{5}{|c|}{ ¿Cómo son sus molestias? } \\
\hline & & & $\begin{array}{l}\text { No siente } \\
\text { molestia } \\
(0)\end{array}$ & $\begin{array}{l}\text { Leve } \\
(1)\end{array}$ & $\begin{array}{l}\text { Moderada } \\
\quad(2)\end{array}$ & $\begin{array}{l}\text { Severo } \\
(3)\end{array}$ & $\begin{array}{l}\text { Muy } \\
\text { severo } \\
\text { (4) }\end{array}$ \\
\hline \multirow{4}{*}{ 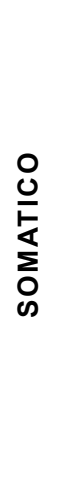 } & 1 & Bochornos o sofocos, sudoración (episodios de sudoración). & & & & & \\
\hline & 2 & $\begin{array}{l}\text { Molestias al corazón (sentir latidos del corazón, palpitaciones, } \\
\text { opresión en el pecho). }\end{array}$ & & & & & \\
\hline & 3 & $\begin{array}{l}\text { Dificultades en el sueño (dificultad para conciliar el sueño, } \\
\text { dificultad para dormir toda la noche, duerme poco, se despierta } \\
\text { muy temprano). }\end{array}$ & & & & & \\
\hline & 11 & $\begin{array}{l}\text { Molestias musculares y articulares (dolores de huesos y } \\
\text { articulaciones, dolores reumáticos). }\end{array}$ & & & & & \\
\hline \multirow{4}{*}{ 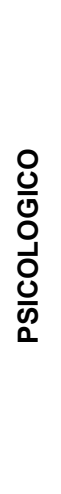 } & 4 & $\begin{array}{l}\text { Estado de ánimo depresivo (sentirse deprimida, decaída, triste, } \\
\text { a punto de llorar, sin ganas de vivir). }\end{array}$ & & & & & \\
\hline & 5 & $\begin{array}{l}\text { Irritabilidad (sentirse tensa, explota fácil, sentirse rabiosa, } \\
\text { sentirse intolerante). }\end{array}$ & & & & & \\
\hline & 6 & $\begin{array}{l}\text { Ansiedad (sentirse angustiada, temerosa, inquieta, tendencia al } \\
\text { pánico). }\end{array}$ & & & & & \\
\hline & 7 & $\begin{array}{l}\text { Cansancio físico y mental (disminución general del rendimiento, } \\
\text { olvidos frecuentes, falta de memoria, le cuesta concentrarse). }\end{array}$ & & & & & \\
\hline \multirow{3}{*}{ 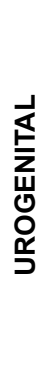 } & 8 & $\begin{array}{l}\text { Problemas sexuales (cambios en el deseo sexual, menor } \\
\text { frecuencia de relaciones sexuales, menos satisfacción sexual). }\end{array}$ & & & & & \\
\hline & 9 & $\begin{array}{l}\text { Problemas de la vejiga (problemas al orinar, orina más veces, } \\
\text { urgencia de orinar, se le escapa la orina). }\end{array}$ & & & & & \\
\hline & 10 & $\begin{array}{l}\text { Sequedad vaginal (sensación de genitales secos, malestar } \\
\text { o ardor en genitales, malestar o dolor con las relaciones } \\
\text { sexuales). }\end{array}$ & & & & & \\
\hline & & PUNTUACION TOTAL & & & & & \\
\hline
\end{tabular}




\section{REFERENCIAS BIBLIOGRÁFICAS}

1. Blumel JE, Lavín P, Vallejo MS, Sarrá S. Menopause or climacteric, just a semantic discussion or has

it clinical implications?. Climacteric 2014 Jun;17(3):235-41.

2. Pines A, Sturdee DW, Mac Lennan $H$. Quality of life and the role of menopausal hormone

therapy. Climacteric 2012;15:213-216.

3. WHOQOL Group. The World Health Organization Quality of Life Assessment (WHOQOL): Position Paper from the World Health Organization. Soc Sci Med 1995;41(10):1403-9.

4. Bullinger $\mathrm{M}$, Anderson $\mathrm{R}$, Cella $\mathrm{D}$, Aranson $\mathrm{N}$. Developing and evaluating cross-cultural instruments from minimun requirements to optimal models. Qual Life Res 1993;2(6):451-9.

5. Siseles N, Pecci C, Mir Candal L, et al. Impacto de la menopausia sobre la calidad de vida y expectativa de vida de la mujer en la mediana edad. En: Siseles $\mathrm{N}$ y cols. Información y Formación para el Manejo Actual de la Mujer en su Climaterio ( $1^{\circ}$ edición). Buenos Aires: Ascune Hnos.2005; 41-64.

6. Siseles N, Gutierrez P, Mir L. Calidad de vida de la mujer en el transcurso del climaterio. En: Artega E, Bianchi M. Climaterio y Menopausia en el Siglo XXI. $1^{\circ}$ edición. Editorial Maval Ltda. Santiago de Chile 2011:103-112.

7. Burger HG, Dudley EC, Robertson DM, Dennerstein L. Hormonal changes in the menopause transition. Recent Prog Horm Res 2002; 57: 257-75.

8. Buckler $\mathrm{H}$. The menopause transition: endocrine changes and clinical symptoms. J Br Menopause Soc 2005; 11: 61-5.

9. Guyatt $\mathrm{GH}$, Veldhuyzen Van Zanten SJ, et al. Measuring quality of life in clinical trials:A taxonomy and review. CMAJ 1989;140(12):1441-8.

10. Permanyer G, Alonso J. La medición del estado de salud en cardiología. Med Cli (Barcelona) 1991; 97:456-8.

11. Palacios S. Instrumentos para medir calidad de vida en la posmenopausia. En: Arteaga E, Bianchi M. Climaterio y menopausia en el Siglo XXI. Tangram Ediciones. Santiago de Chile 2011; pp:94-102

12. Wiklund I, Dimenas E, Wahl M. Factors of importance when evaluating quality of life in clinical trials. Control Clin Trials 1990;11:169-79.

13. Ferrer Barrientos J. Tipos de escala de calidad de vida:¿tenemos lo que necesitamos? VII congreso de la Asociación Española para el estudio de la Menopausia, Cadiz, 14-17 de mayo de 2002, Libro de resúmenes 118-121.

14. McKenna S, Hunt S, Tennant A. The development of a patient completed index of distress from the Nottingham Health Profile:a new measure for use in cost-utitity analysis. $20 \mathrm{Br} \mathrm{J}$ Med Economics 1993;6:13-24.

15. Ware I, Sherbourne C. The MOS 36-item short-form survey (SF-36). Med Care 1992;30:473-83.

16. Dupuy $H$. The psychological general well-being index. In: Wenger N, Mattson M, Furberg C, et al. Assessment of quality of life in Clinical Trials of Cardiovascular Therapies. USA. Le Jacq 1984;170-83.

17. Badía X, Roset M, Montserrat S, et al. La version española del Euo QoL: descripción y aplicaciones. Med Clin 1999;112 (suppl 1):79-85

18. Ledésert B, Ringa $V$, Bréart $G$. Menopause and perceived health status among the women of the French GAZEL cohort. Maturitas 1995;20:113-20.

19. McHorney C, Ware J, Lu J, et al. The MOS 36-ítem ShortForm Health Survey (SF-36): III. Tests of data quality, scaling assumptions, and reliability across diverse patient groups. Med Care 1994;32:40-66.

20. Apolone G, Mosconi P. The Italian SF-36 Health Survey: translation, validation and norming. $\mathrm{J}$ Clin Epidemiol 1998;51:1025-36.

21. O'Dea L, Hunter S, Anjos S. Life satisfaction and healthrealted quality of life (SF-36) of middle-aged men and women. Climateric 1999;2:131-40.

22. Ferrer J, Valdés D. Utilidad de la medición de la calidad de vida en el climaterio. Rev Iberoam Menop 2005; 7(1):4-8.

23. Utian W, Yu H, Bobula J, et al. Bazedoxifene/conjugated estrogens and quality of life in postmenopausal women. Maturitas 2009; 63(4):329-35.

24. Kupperman HS, Blatt MH, Wiesbader $\mathrm{H}$, et al. Comparative clinical evaluation of estrogenis preparations by the menopausal and amenorrheal indices. J Clin Endocrinol Metab 1953; 13:688-703.

25. Hunter M. The Women's Health Questionnaire: a measure of mid-aged women's perceptions of their emotional and physical health. Psychol Health 1992; 7: 45-54.

26. Buendía J, Rodríguez R, Yubero N, y col. Diseño y validación de un cuestionario en castellano para medir calidad de vida en mujeres posmenopáusicas: el cuestionario MENCAV. Atención Primaria 2001; 27(2):94-100.

27. Scheneider HP, Heinemann LA, Rosemeier HP, et al. The Menopause Rating Scale (MRS) comparison with Kupperman index and quality of life scale SF-36. Climateric 2000; 3:50-8.

28. Hilditch JR, Lewis J, Peter A, et al. A menopause specific quality of life questionnaire, development and psychometric properties. Maturitas 1996; 24: 161-75.

29. Blumel JE, Castelo-Branco C, Binfa L, et al. Quality of life after the menopause: a population study. Maturitas 2000; 34: 17-23.

30. Utian WH, Janata JW, Kingsberg SA, et al. The Utian Quality of Life (UQOL) Scale: development and validation of an instrument to quantify quality of life through and beyond menopause. Menopause 2002; 9: 402-10.

31. Greene JG. Constructing a standard climateric scale. Maturitas 29(1998):25-31

32. Palacios S, Ferrer-Barrientos J, Parrilla JJ, et al. Calidad de vida relacionada con la salud de la mujer española durante la perimenopausia y postmenopausia. Desarrollo y validación de la Escala Cervantes. Med Clin 2004; 122(6): 205-11.

33. Alder E. The Blatt Kupperman Menopausal index: a critique. Maturitas 1998; 29:19-24

34. Ortiz N, Mariño C, Méndez B, Olarte N. Tesis de Epidemiología: influencia del climaterio en la calidad de vida. Fac. Medicina. Colegio Mayor de Nuestra señora del Rosario. Santa Fé de Bogotá. Colombia 2000.

35. Ayala-Peralta FD, Muñoz R, Celedonio M, Rivera J, Ayala R. Calidad de vida en climaterio. Rev Per Ginecol Obstet 2003;49:160-5.

36. Sierra B, Hidalgo LA, Chedraui PA. Measuring climacteric symptoms in an Ecuadorian population with the Greene Climacteric Scale. Maturitas 2005;51:236-245.

37. Ayala-Peralta F. Influencia del climaterio en la calidad de vida relacionada a la salud femenina en el Instituto Nacional Materno Perinatal. Tesis de Magíster. Facultad de Medicina Humana. Universidad Nacional Mayor de San Marcos, 2008. Lima-Perú.

38. Hauser G, Huber I, Keller P, et al. The Menopause Rating Scale (MRS). Zentralbl Gynakol 1994; 116:16-23.

39. Schneider $H$, Doren $M$. Traits for long-term acceptance of hormone replacement therapy results of a representative German survey. Eur Menopause J 1996; 3:94-8. 
40. Schneider HP, Heinemann LA, Rosemeier HP, et al. The Menopause Rating Scale (MRS): reliability of scores of menopausal complaints. Climateric 2000;3:59-64.

41. Hauser GA: A new rating scale for the climacteric syndrome (Menopause Rating Scale (MRS). Schweiz Med Wochenschr 1997, 127: 122-127.

42. Chedraui P, Blümel JE, Baron G, Belzares E, Bencosme A, Calle A, Dancker L, Izaguirre $H$, et al. Impaired quality of life among middle aged women: A multicentre Latin American study. Maturitas 2008;61:323-329.

43. Monterrosa A, Blumel JE, Chedraui P. Increased menopausal symptoms among Afro-Colombian women as assessed with the Menopause Rating Scale. Maturitas 2008;59:182-190.

44. Monterrosa A, Blumel JE, Chedraui P. Calidad de vida de mujeres en postmenopausia. Valoración con "Menopause Rating Scale" de tres etnias colombianas diferentes. Med UNAB 2009; 12: 80-85.

45. Ojeda E, Monterrosa A, Blümel JE, Escobar-López J, Chedraui P. Severe menopausal symptoms in mid-aged Latin American women can be related to their indigenous ethnic component. Climacteric 2011;14:157-163.

46. Chedraui P, San Miguel G, Avila C. Quality of life impairment during the female menopausal transition is related to personal and partner factors. Gynecol Endocrinol 2009;25:130-135.

47. Binfa L, Castelo-Branco C, Blumel Je, Cancelo Mj, Bonilla $\mathrm{H}$, Munoz I Et Al. Influence of psycho-social factors on climacteric symptoms. Maturitas 2004;48: 425-31.

48. Blumel JE, Castelo-Branco C, Cancelo MJ, Cordova AT, Binfa LE, Bonilla HG, et al. Relationship between psychological complaints and vasomotor symptoms during climacteric. Maturitas 2004; 49: 205-10.

49. Chedraui P, Aguirre W, Hidalgo L, Fayad L. Assessing menopausal symptoms among healthy middle aged women with the Menopause Rating Scale. Maturitas 2007; 57: 271-8.
50. Appling S, Paez K, Allen J. Ethnicity and vasomotor symptoms in postmenopausal women. J Women's Health (Larchmt) 2007; 16:1130-8.

51. Sievert LL, Morrison L, Brown DE, Reza AM. Vasomotor symptoms among Japanese-American and EuropeanAmerican women living in Hilo, Hawaii. Menopause 2007; 14:261-9

52. Gold EB, Sternfeld B, Kelsey JL, Brown C, Mouton C, et al. Relation of demographic and lifestyle factors to symptoms in a multi-racial/ethnic population of women 40-55 years of age. Am J Epidemiol 2000; 152: 463-73.

53. Siseles N, Gutierrez P, Sayegh F. The climateric in Latin America: Actual state and future trends. In: The Menopause at the Millennium. The proceedings of the $9^{\text {th }}$ International Menopause Society World Congress on the Menopause. Yokohama, Japan. The Parthenon Publishing Group New York, London; 2000:108-116.

54. Oldehave A, Jaszmann LJ, Haspels AA, et al. Impact of climacteric on well-being. Am J Obstet Gynecol 1996; 168: 772-80.

55. Bachmann G, Ebert G, Burd I. Vulvovaginal complaints In: Treatment of the postmenopausal women: basic and clinical aspects, $2^{\text {nd }}$ edition. Lobo R, Lippincott Williams \& Williams, Philadelphia 1999;18:195-201.

56. Dennerstein L, Smith A, Morse C. Psychological well-being, mild-life and the menopause. Maturitas 1994;20:1.

57. Utian W. Rol de la hormonoterapia a largo plazo en el contexto de la medicina preventiva para la mujer postmenopáusica. En: Gonzales O, Arteaga E y Contreras P. Menopausia y patologías asociadas. Santiago de Chile: ediciones Sociedad Chilena de Climaterio; 1998:25-33. 